\title{
Dynamical Generation of Elementary Fermion Mass: First Lattice Evidence
}

\author{
S. Capitani, ${ }^{1}$ P. Dimopoulos, ${ }^{2,3}$ R. Frezzotti,${ }^{3,4,{ }^{*}}$ M. Garofalo, ${ }^{4}$ B. Kostrzewa, ${ }^{5}$ F. Pittler, ${ }^{5}$ \\ G. C. Rossi, ${ }^{2,3,4}$ and C. Urbach ${ }^{5}$ \\ ${ }^{1}$ Goethe U. Frankfurt, Inst. f. Theor. Phys, Max-von-Laue-Str. 1, 60438 Frankfurt am Main, Germany \\ ${ }^{2}$ Centro Fermi-Museo Storico della Fisica e Centro Studi e Ricerche Enrico Fermi, 00184 Rome, Italy \\ ${ }^{3}$ Dipartimento di Fisica, Università di Roma Tor Vergata, Via della Ricerca Scientifica, O0133 Roma, Italy \\ ${ }^{4}$ INFN, Sezione di Roma Tor Vergata, Via della Ricerca Scientifica, 00133 Roma, Italy \\ ${ }^{5}$ HISKP (Theory), Rheinische Friedrich-Wilhelms-Universität Bonn, Nussallee 14-16, 53115 Bonn, Germany
}

(Received 8 November 2018; revised manuscript received 28 May 2019; published 9 August 2019)

\begin{abstract}
Using lattice simulations we demonstrate from first principles the existence of a nonperturbative mechanism for elementary particle mass generation in models with gauge fields, fermions, and scalars, if an exact invariance forbids power divergent fermion masses and fermionic chiral symmetries broken at UV scale are maximally restored. We show that in the Nambu-Goldstone phase a fermion mass term, unrelated to the Yukawa operator, is dynamically generated. In models with electroweak interactions weak boson masses are also generated, opening new scenarios for beyond the standard model physics.
\end{abstract}

DOI: 10.1103/PhysRevLett.123.061802

Introduction.-In spite of its impressive phenomenological success, the standard model (SM) of particle physics is believed to represent only an effective low energy theory, as it neither accounts for dark matter and quantum aspects of gravity nor provides enough $C P$ violation for baryogenesis. Fermion and electroweak (EW) boson masses are described in terms of a wellestablished symmetry breaking pattern [1], but the SM is by construction unable to shed light on the problems of EW scale naturalness [2] and fermion mass hierarchy [3].

There have been numerous attempts to build phenomenologically viable models where the EW scale is stable under quantum corrections, either because the basic theory enjoys an approximate symmetry larger than in the SM or because EW and Higgs mass scales are related in a fixed way to a fundamental dynamical scale. Examples of the first kind are the many models based on supersymmetry (SUSY) [4,5] which, besides having the problem of explaining SUSY breaking, are presently disfavored owing to the experimental exclusion of SUSY particles with mass up to a few TeV [6]. Approaches of the second kind assume the existence of some new interaction that gets strong around or above the EW scale, and of new fermionic particles. The original technicolor idea $[7,8]$ could account for the EW boson masses, but attempts to understand heavy fermion masses in extended technicolor (ETC)

Published by the American Physical Society under the terms of the Creative Commons Attribution 4.0 International license. Further distribution of this work must maintain attribution to the author(s) and the published article's title, journal citation, and DOI. Funded by SCOAP . models $[9,10]$ face severe problems to comply with experimental constraints on flavor changing neutral currents, even in subsequently developed walking ETC models [11-14]. Other ways to comply with experimental constraints and possibly address the flavor hierarchy problem are represented by the partially composite Higgs models $[15,16]$ and by models with extra dimensions [17-19].

At variance with previous attempts, a novel intrinsically nonperturbative (NP) mechanism for elementary fermion mass generation was conjectured in Ref. [20]. This mechanism is expected to be at work in non-Abelian gauge models where (as usual) (i) chiral transformations acting on fermions and scalars are exact symmetries, but (deviating from common assumptions) (ii) purely fermionic chiral symmetries undergo an explicit breaking at the UV scale. When bare parameters are "naturally" tuned so as to minimize fermion chiral breaking, in the effective Lagrangian (EL-generating functional of proper vertices) no Yukawa term occurs, but operators of NP origin that violate fermion chiral symmetries, among which a fermion mass term, should appear, if the scalar potential is such that the theory lives in its Nambu-Goldstone (NG) phase. Upon introducing $\mathrm{EW}$ interactions, the same mechanism also yields massive $W^{ \pm}, Z^{0}$ bosons and a composite Higgs boson in the $W^{+} W^{-} / Z^{0} Z^{0}$ channel [21].

In this Letter we employ lattice simulations (lacking analytical methods) to provide evidence from first principles for the occurrence of the NP mass generation mechanism of Ref. [20], within the simplest $d=4$ model where it could take place.

Mass generation in a toy model.-The Lagrangian of the "toy" (yet nontrivial) model of interest here [20] reads 


$$
\mathcal{L}_{\text {toy }}=\mathcal{L}_{k}(Q, A, \Phi)+\mathcal{V}(\Phi)+\mathcal{L}_{W}(Q, A, \Phi)+\mathcal{L}_{Y}(Q, \Phi)
$$

with $\mathcal{L}_{k}$ and $\mathcal{V}$ representing standard kinetic terms and scalar potential. $\mathcal{L}_{\text {toy }}$ includes an $\mathrm{SU}(3)$ gauge field, $A_{\mu}$, with bare (renormalized) coupling $g_{0}\left(g_{s}\right)$, a Dirac doublet, $Q=(u, d)^{T}$, transforming as a triplet under $\mathrm{SU}(3)$ and a complex scalar doublet, $\varphi=\left(\varphi_{0}+i \varphi_{3},-\varphi_{2}+i \varphi_{1}\right)^{T}$, singlet under SU(3). For the latter we use the $2 \times 2$ matrix notation $\Phi=\left[\varphi \mid-i \tau^{2} \varphi^{*}\right]$. The model has an UV cutoff $\Lambda_{\mathrm{UV}} \sim b^{-1}$ and includes a Yukawa term, $\mathcal{L}_{Y}(Q, \Phi)=$ $\eta\left(\bar{Q}_{L} \Phi Q_{R}+\bar{Q}_{R} \Phi^{\dagger} Q_{L}\right)$, as well as a nonstandard term

$\mathcal{L}_{W}(Q, A, \Phi)=\frac{b^{2}}{2} \rho\left(\bar{Q}_{L} \overleftarrow{\mathcal{D}}_{\mu} \Phi \mathcal{D}_{\mu} Q_{R}+\bar{Q}_{R} \overleftarrow{\mathcal{D}}_{\mu} \Phi^{\dagger} \mathcal{D}_{\mu} Q_{L}\right)$.

The latter is a $\Lambda_{\mathrm{UV}}^{-2} \times d=6$ operator that leaves the model power-counting renormalizable [20], like it happens for the Wilson term in lattice QCD [22,23]. Neither $\mathcal{L}_{W}$ nor $\mathcal{L}_{Y}$ are invariant under purely fermionic chiral transformations.

Among other symmetries, the Lagrangian (1) is invariant under the global transformations $\left[\Omega_{L / R} \in \mathrm{SU}(2)\right]$

$$
\begin{aligned}
& \chi_{L} \times \chi_{R}=\left[\tilde{\chi}_{L} \times\left(\Phi \rightarrow \Omega_{L} \Phi\right)\right] \times\left[\tilde{\chi}_{R} \times\left(\Phi \rightarrow \Phi \Omega_{R}^{\dagger}\right)\right], \\
& \tilde{\chi}_{L / R}: Q_{L / R} \rightarrow \Omega_{L / R} Q_{L / R}, \quad \bar{Q}_{L / R} \rightarrow \bar{Q}_{L / R} \Omega_{L / R}^{\dagger} .
\end{aligned}
$$

No power divergent fermion masses can be generated as a term like $\Lambda_{\mathrm{UV}}\left(\bar{Q}_{L} Q_{R}+\bar{Q}_{R} Q_{L}\right)$ is not $\chi_{L} \times \chi_{R}$ invariant.

Wigner phase and fermion chirality restoration.- $\mathcal{L}_{\text {toy }}$ is not invariant under the purely fermionic chiral transformations $\tilde{\chi}_{L} \times \tilde{\chi}_{R}$. However, as shown in [20], in the phase with positive renormalized squared scalar mass $\left(\hat{\mu}_{\phi}^{2}>0\right)$, where the $\chi_{L} \times \chi_{R}$ symmetry is realized à la Wigner, a critical value of the Yukawa coupling, $\eta_{c r}$, exists at which [up to $O\left(b^{2}\right)$ corrections] the effective Yukawa term vanishes. The renormalized Schwinger-Dyson equations (SDE), say for the $\tilde{\chi}_{L}$ transformations read (no sum over $i=1,2,3$, $|x| \gg b)$

$$
\begin{aligned}
\partial_{\mu}\left\langle Z_{\tilde{J}} \tilde{J}_{\mu}^{L i}(x) \hat{O}^{i}(0)\right\rangle & =(\bar{\eta}-\eta)\left\langle\left(\tilde{D}_{L}^{i}(x) \hat{O}^{i}(0)\right\rangle+\mathrm{O}\left(b^{2}\right),\right. \\
\tilde{D}_{L}^{i} & =\bar{Q}_{L} \frac{\tau^{i}}{2} \Phi Q_{R}-\bar{Q}_{R} \Phi^{\dagger} \frac{\tau^{i}}{2} Q_{L},
\end{aligned}
$$

with $\hat{O}^{i}$ any $\tilde{\chi}_{L}$ covariant operator. The current $\tilde{J}_{\mu}^{L i}$ is given in Ref. [20]. Owing to parity, similar SDE hold for $\tilde{\chi}_{R}$. At the value $\eta=\eta_{c r}\left(g_{0}^{2}, \rho, \lambda_{0}\right)$ that solves the equation $\eta-\bar{\eta}\left(\eta ; g_{0}^{2}, \rho, \lambda_{0}\right)=0$ the SDE take the form of WardTakahashi identities (WTI) and the fermionic chiral transformations $\tilde{\chi}_{L} \times \tilde{\chi}_{R}$ become symmetries [24] of the model (1) up to $O\left(b^{2}\right)$ terms. In Eq. (5) the dimensionless coefficient $\bar{\eta}=\bar{\eta}\left(\eta ; g_{0}^{2}, \rho, \lambda_{0}\right)$ stems from the mixing of the $\tilde{\chi}_{L}$ variations of $\mathcal{L}_{W}$ and $\mathcal{L}_{Y}$, while $Z_{\tilde{J}}$ is a multiplicative renormalization factor (free of logarithmic UV divergencies at $\eta=\eta_{\mathrm{cr}}$ ). Symmetries constrain the expression of the EL so that in the Wigner phase its $d=4$ piece is analogous in form to $\mathcal{L}_{\text {toy }}$, namely,

$$
\begin{aligned}
\Gamma_{4}^{W i g} \equiv & \Gamma_{\hat{\mu}_{\phi}^{2}>0} \\
= & \Gamma_{k}(A, Q, \Phi) \\
& +\left[\eta-\bar{\eta}\left(\eta ; g_{0}^{2}, \rho, \lambda_{0}\right)\right]\left(\bar{Q}_{L} \Phi Q_{R}+\bar{Q}_{R} \Phi^{\dagger} Q_{L}\right) \\
& +\hat{\mathcal{V}}(\Phi), \\
\Gamma_{k}=\frac{1}{4} & (F F)+\bar{Q}_{L} \not D Q_{L}+\bar{Q}_{R} \not D Q_{R}+\frac{1}{2} \operatorname{Tr}\left[\partial_{\mu} \Phi^{\dagger} \partial_{\mu} \Phi\right] .
\end{aligned}
$$

The Wigner phase is thus well suited to determine the critical value of $\eta$, where the effective Yukawa term disappears from Eq. (7). We stress that, neglecting $O\left(b^{2}\right)$ artifacts, $\bar{\eta}$ and $\eta_{\mathrm{cr}}$ are independent of the subtracted scalar mass $\hat{\mu}_{\phi}^{2}$ (see Supplemental Material [25], Sec. III) and thus equal in the Wigner and NG phase. From Eq. (5) $\eta_{\text {cr }}$ can be determined, e.g., as the value of $\eta$ where (no sum over $i=1,2,3, x \neq 0$ )

$$
\begin{aligned}
& \frac{\partial_{\mu}\left\langle\tilde{A}_{\mu}^{i}(x) \tilde{D}_{P}^{i}(0)\right\rangle}{\left\langle\tilde{D}_{P}^{i}(x) \tilde{D}_{P}^{i}(0)\right\rangle}=0, \quad \tilde{A}_{\mu}^{i}=\tilde{J}_{\mu}^{L i}-\tilde{J}_{\mu}^{R i}, \\
& \tilde{D}_{P}^{i}=\bar{Q}_{L}\left\{\Phi, \frac{\tau^{i}}{2}\right\} Q_{R}-\bar{Q}_{R}\left\{\frac{\tau^{i}}{2}, \Phi^{\dagger}\right\} Q_{L} .
\end{aligned}
$$

An equivalent, but statistically less noisy, condition is discussed below (Lattice study and results). We stress that the existence of an $\eta_{\mathrm{cr}}$, where the $\tilde{\chi}_{L} \times \tilde{\chi}_{R}$ transformations become symmetries of the theory [up to $O\left(b^{2}\right)$ ], is a general property of the Wigner phase independently of the specific form of $\mathcal{L}_{W}$. Owing to renormalizability and universality, changing $\mathcal{L}_{W}$ would only modify the values of $\eta_{\mathrm{cr}}, Z_{\tilde{J}}$, and $O\left(b^{2}\right)$ artifacts.

Nambu-Goldstone phase and NP anomaly.-Most interesting is the case $\hat{\mu}_{\phi}^{2}<0$, where $\mathcal{V}(\Phi)$ has a double-well shape with $\left\langle: \Phi^{\dagger} \Phi:\right\rangle=v^{2} 1, v \neq 0$, so that the $\chi_{L} \times \chi_{R}$ symmetry is realized in the manner of NG. In large volume under any, even infinitesimal, symmetry breaking perturbation the $\chi_{L} \times \chi_{R}$ symmetry will be spontaneously broken to $\mathrm{SU}(2)_{V}$. Moreover, at $\eta=\eta_{\text {cr }}$ residual $\mathrm{O}\left(b^{2} v\right) \tilde{\chi}_{L} \times \tilde{\chi}_{R}$ violating terms will polarize the degenerate vacuum resulting from the spontaneous $\tilde{\chi}_{L} \times \tilde{\chi}_{R}$ symmetry breaking brought about by strong interactions.

Realization of the $\chi_{L} \times \chi_{R}$ invariance in the manner of NG has a key impact on low energy physics. Three elementary massless Goldstone bosons appear in the spectrum which need to be included in the EL. In Ref. [20] it was argued that at $\eta=\eta_{c r}$ the EL describing the model (1) should include $\tilde{\chi}_{L} \times \tilde{\chi}_{R}$ violating terms of NP origin, among which a fermion mass term. 
This conjecture can be checked by studying the SDE associated with the $\tilde{\chi}_{L} \times \tilde{\chi}_{R}$ transformations [see, e.g., Eq. (5)] in the NG phase. Exploiting parity invariance, we more conveniently evaluate the effective PCAC mass from the axial SDE (no sum over $i=1,2,3, x_{0} \gg b$ )

$\left.\frac{Z_{\tilde{A}}}{Z_{P}} m_{\mathrm{AWI}} \equiv \frac{Z_{\tilde{A}} \sum_{\mathbf{x}} \partial_{0}\left\langle\tilde{A}_{0}^{i}(x) P^{i}(0)\right\rangle}{2 Z_{P} \sum_{\mathbf{x}}\left\langle P^{i}(x) P^{i}(0)\right\rangle}\right|_{\eta_{\mathrm{cr}}}, \quad P^{i}=\bar{Q} \gamma_{5} \frac{\tau^{i}}{2} Q$,

where $Z_{\tilde{A}}=\left.Z_{\tilde{J}}\right|_{\eta_{c r}}$ and $Z_{P}$ are renormalization factors. If $\mathrm{NP} \tilde{\chi}_{L} \times \tilde{\chi}_{R}$ violating terms were absent in the critical EL, one should find $\left(Z_{\tilde{A}} / Z_{P}\right) m_{\mathrm{AWI}} \rightarrow 0$ as $b \sim \Lambda_{\mathrm{UV}}^{-1} \rightarrow 0$. Lattice simulations (see Lattice study and results) show that $\left(Z_{\tilde{A}} / Z_{P}\right) m_{\mathrm{AWI}}$ is not vanishing in the continuum limit.

In the NG phase the EL describing the model (1) is expressed in terms of effective fermion, gauge, and scalar fields. The latter, in view of $v \neq 0$, are conveniently rewritten [60] introducing Goldstone $\left(\zeta_{1,2,3}\right)$ and massive $\left(\zeta_{0}\right)$ scalar fields in the form

$$
\Phi=R U, \quad R=\left(v+\zeta_{0}\right), \quad U=\exp \left[i v^{-1} \tau^{k} \zeta_{k}\right],
$$

where $U$ is a dimensionless field transforming as $U \rightarrow$ $\Omega_{L} U \Omega_{R}^{\dagger}$ under $\chi_{L} \times \chi_{R}$. The nonanalytic field $U$ only makes sense if $v \neq 0$. At $\eta=\eta_{c r}$ the $d \leq 4$ EL sector reads [20] [see Eq. (7)]

$$
\begin{aligned}
\Gamma_{4}^{\mathrm{NG}}= & c_{2} \Lambda_{S}^{2} \operatorname{Tr}\left(\partial_{\mu} U^{\dagger} \partial_{\mu} U\right)+c_{1} \Lambda_{S}\left[\bar{Q}_{L} U Q_{R}+\text { H.c. }\right] \\
& +\tilde{c} \Lambda_{S} R \operatorname{Tr}\left(\partial_{\mu} U^{\dagger} \partial_{\mu} U\right)+\Gamma_{\hat{\mu}_{\phi}^{2}<0}+\mathrm{O}\left(\Lambda_{S}^{2} / v^{2}\right),
\end{aligned}
$$

where $\Lambda_{S}$ is the renormalization group invariant (RGI) scale and the term $\propto c_{1}$ describes the NP breaking of $\tilde{\chi}_{L} \times \tilde{\chi}_{R}$. Upon expanding $U$ around the identity one gets

$c_{1} \Lambda_{S}\left[\bar{Q}_{L} U Q_{R}+\bar{Q}_{R} U^{\dagger} Q_{L}\right]=c_{1} \Lambda_{S} \bar{Q} Q[1+\mathrm{O}(\zeta / v)]$,

thus a fermion mass term plus a host of more complicated, nonpolynomial $\bar{Q}-\zeta_{1,2,3}{ }^{\prime} s-Q$ vertices.

The NP term (14) was conjectured in Ref. [20] to arise dynamically in the EL (13) of the theory (1) from the interplay of strong interactions and the breaking of $\tilde{\chi}_{L} \times \tilde{\chi}_{R}$ at the UV scale. The occurrence of this $\tilde{\chi}_{L} \times \tilde{\chi}_{R}$ violating term in the EL is essential to account for the nonzero value we find for $\left.Z_{\tilde{A}} \sum_{\mathbf{x}} \partial_{0}\left\langle\tilde{A}_{0}^{i}(x) P^{i}(0)\right\rangle\right|_{\eta=\eta_{\text {cr }}}$ [Eq. (11)] despite the fact that the operators $\tilde{A}_{0}^{i}$ and $P^{i}$ transform differently under $\tilde{\chi}_{L} \times \tilde{\chi}_{R}$. The coefficient $c_{1}$ in Eq. (14) has been argued in Ref. [20] to be an $O\left(g_{s}^{4}\right)$ odd function of $\rho$. As for its dependence on the scalar squared mass, $c_{1}$ is expected to stay finite in the phenomenologically interesting limit $-\hat{\mu}_{\phi}^{2} \gg \Lambda_{S}^{2}[20,21]$ and be nonzero only for $\hat{\mu}_{\phi}^{2}<0$. Indeed in our interpretation the NP term (14) arises from the spontaneous $\tilde{\chi}_{L} \times \tilde{\chi}_{R}$ breaking which is effective only in the NG phase where the degenerate vacuum gets polarized by residual $O\left(b^{2} v\right) \tilde{\chi}_{L} \times \tilde{\chi}_{R}$ breaking effects.

A proper understanding of the NP terms in the expression (13) requires considering what is the natural extension of the $\tilde{\chi}_{L} \times \tilde{\chi}_{R}$ symmetry in the presence of EW interactions [21]. In that context one finds that maximal restoration of the chiral fermion symmetry entails the vanishing of the coefficient $\tilde{c}$ in Eq. (13), leaving only the NP terms with coefficients $c_{1}$ and $c_{2}$, which are responsible for the dynamical mass of fermions and weak bosons, respectively [20,21].

At $\eta=\eta_{\mathrm{cr}}$ all the NP terms occurring in the right-hand side of the $\tilde{\chi}_{L} \times \tilde{\chi}_{R}$ SDE must be RGI, as the left-hand side of the SDE is. Conservation up to $O\left(b^{2}\right)$ of the $Z_{\tilde{J}} \tilde{J}_{\mu}^{L(R) i}$ currents makes the left-hand side of the $\tilde{\chi}_{L} \times \tilde{\chi}_{R}$ SDE scale invariant independently of $\hat{\mu}_{\phi}^{2}$.

The full NG phase EL, $\Gamma^{N G} \supset \Gamma_{4}^{N G}$ contains of course an infinite number of local terms of arbitrarily high dimension, among which terms of NP origin that break the $\tilde{\chi}_{L} \times \tilde{\chi}_{R}$ symmetry. The occurrence of these NP and RGI terms in the EL will be referred to as a "NP anomaly" in the restoration of $\tilde{\chi}_{L} \times \tilde{\chi}_{R}$ symmetry.

Lattice study and results.-We describe the main steps and results of our numerical study of the model (1) in a lattice regularization consistent with the exact $\chi_{L} \times \chi_{R}$ symmetry. Lattice $\chi_{L} \times \chi_{R}$ invariance entails relations between renormalization constants (e.g., $Z_{\tilde{A}}=Z_{\tilde{V}}$ ) and discretization errors of $O\left(b^{2 n}\right)$ only ( $n$ integer). The arguments of Ref. [20] imply that the NP anomaly leading to elementary fermion mass generation occurs even if fermion loops are neglected. Thus in this first investigation we decided to work in the quenched approximation [61]. Quenching brings key simplifications as scalar and gauge fields can be generated and renormalized independently from each other. In the Supplemental Material [25] we give details on our lattice setup and data analysis.

For a given choice of bare gauge coupling $\left(\beta=6 / g_{0}^{2}\right)$, scalar potential parameters $\left(\lambda_{0}, m_{\phi}^{2}-m_{c r}^{2}=\hat{\mu}_{\phi}^{2} / Z_{m_{\phi}^{2}}\right)$ and strength $(\rho)$ of the $\mathcal{L}_{W}$ term, we tune the bare coupling $\eta$, so as to restore the fermionic chiral symmetry $\tilde{\chi}_{L} \times \tilde{\chi}_{R}$. This task is conveniently carried out in the Wigner phase by looking for the value of $\eta$ at which

$$
\begin{aligned}
& r_{\mathrm{AWI}}\left(\eta_{\mathrm{cr}}, \rho, \lambda_{0}, g_{0}^{2}\right)=\left.\frac{\mathcal{N}\left(x_{0}, y_{0} ; \eta, \rho, \lambda_{0}, g_{0}^{2}\right)}{\mathcal{D}\left(x_{0}, y_{0} ; \eta, \rho, \lambda_{0}, g_{0}^{2}\right)}\right|_{\eta=\eta_{\mathrm{cr}}}=0, \\
& \mathcal{N}\left(x_{0}, y_{0} ; \ldots\right)=b^{6} \sum_{\mathbf{x}, \mathbf{y}}\left\langle P^{1}(0) \partial_{0}^{F W} \tilde{A}_{0}^{1, B W}(x) \varphi_{0}(y)\right\rangle, \\
& \mathcal{D}\left(x_{0}, y_{0} ; \ldots\right)=b^{6} \sum_{\mathbf{x}, \mathbf{y}}\left\langle P^{1}(0) \tilde{D}_{P}^{1}(x) \varphi_{0}(y)\right\rangle, \quad \varphi_{0}=\frac{\operatorname{Tr}[\Phi]}{2},
\end{aligned}
$$




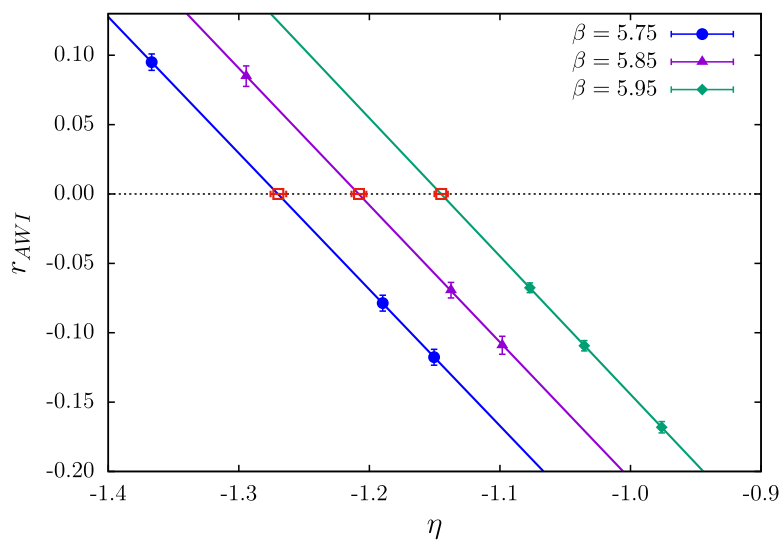

FIG. 1. $\quad r_{\mathrm{AWI}}$ as a function of $\eta$ at $\beta=5.75,5.85$, and 5.95. Red squares denote the values of $\eta_{\mathrm{cr}}$, at which $r_{\mathrm{AWI}}=0$.

where $\partial_{0}^{F W}$ is a forward lattice derivative and $\tilde{A}_{0}^{1, B W}$ the backward one-point-split lattice version of $\bar{Q} \gamma_{0} \gamma_{5}\left(\tau^{1} / 2\right) Q$. $\tilde{D}_{P}^{1}$ and $P^{1}$ are given in Eqs. (10) and (11), respectively. The time distances $x_{0}$ and $y_{0}-x_{0}$ are separately optimized so as to isolate the lowest-lying pseudoscalar (PS) meson and one- $\Phi$ particle states. In Fig. 1 we show $r_{\mathrm{AwI}}$ vs $\eta$ at three values of the bare gauge coupling, $\beta=5.75,5.85$, and 5.95. In the quenched approximation they correspond to lattice spacings of about $0.15,0.12$, and $0.10 \mathrm{fm}$, respectively, if we conventionally assume for the Sommer scale $r_{0}=0.5 \mathrm{fm}$ (as in QCD). The values of $\left.\eta_{c r}\left(6 / \beta, \rho, \lambda_{0}\right)\right|_{\rho=1.96}$ are denoted by red squares.

In the NG phase we work at $\eta=\eta_{\mathrm{cr}}$, taking into account its uncertainty. First we compute the effective PCAC mass (11). For convenience $Z_{P}^{-1}$ is evaluated in a hadronic scheme defined in the Wigner phase by taking $Z_{P}^{-1} r_{0}^{-2}=G_{\mathrm{PS}}^{W}=\left\langle 0\left|P^{1}\right| \mathrm{PS} \text { meson }\right\rangle^{W}$ at the subtraction point, $M_{\mathrm{PS}}$, given by the PS meson mass (see below). As for $Z_{\tilde{A}}$, we exploit the equality $Z_{\tilde{A}}=Z_{\tilde{V}}$, entailed by the $\chi_{L} \times \chi_{R}$ invariance, and evaluate $Z_{\tilde{V}}$ from an exact WTI.

In Fig. 2 we plot the renormalized quantity $2 r_{0} m_{\mathrm{AWI}} Z_{\tilde{V}} Z_{P}^{-1}$ vs $\left(b / r_{0}\right)^{2}$. A linear extrapolation shows that its continuum limit lies about 3 standard deviations away from zero.

Second, as another check that the effective PCAC mass $\left(Z_{\tilde{A}} / Z_{P}\right) m_{\mathrm{AWI}}$ is nonzero, indicating the presence in the EL of a fermion mass term, we have computed from the $\sum_{\mathbf{X}}\left\langle P^{1}(0) P^{1}(x)\right\rangle$ correlator the mass of the lowest lying PS meson. Our data for $r_{0} M_{\mathrm{PS}}$ are plotted in Fig. 3 as a function of $\left(b / r_{0}\right)^{2}$ together with the best fit linear extrapolation to vanishing lattice spacing. The figure shows that the continuum limit of $r_{0} M_{\mathrm{PS}}$ lies above zero by more than 5 standard deviations. On the other hand, if $r_{0} M_{\mathrm{PS}}$ had a vanishing continuum limit, one should see $r_{0}^{2} M_{\mathrm{PS}}^{2}$ approaching zero as $b^{2} \rightarrow 0$ with a $b^{4}$

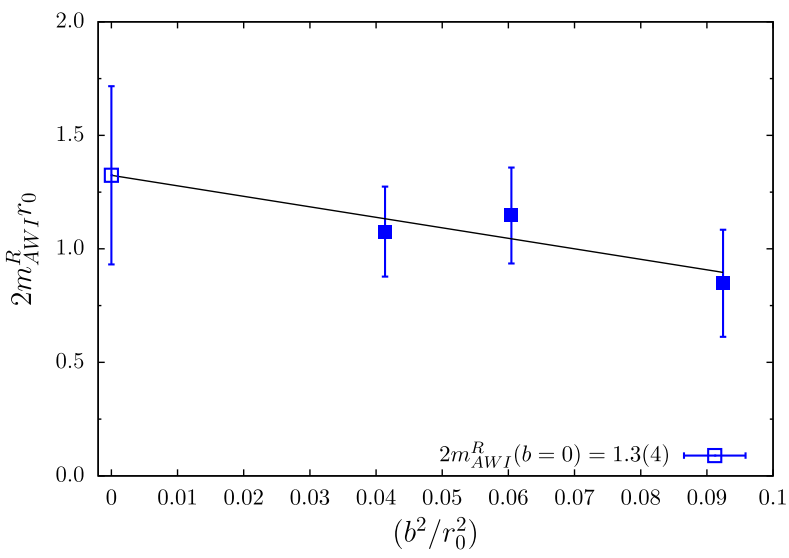

FIG. 2. $2 m_{\mathrm{AWI}}^{R} r_{0} \equiv 2 r_{0} m_{\mathrm{AWI}} Z_{\tilde{V}} Z_{P}^{-1}$ vs $\left(b / r_{0}\right)^{2}$ in the NG phase and its linear extrapolation to the continuum limit.

rate. On a $\left(b / r_{0}\right)^{4}$ scale our $r_{0}^{2} M_{\mathrm{PS}}^{2}$ data lie very close to the continuum limit and are not compatible with a "no mechanism" hypothesis (see Supplemental Material [25]).

Finally, we checked that at $\eta=\eta_{\mathrm{cr}}(\rho)$ the magnitude of $\left(Z_{\tilde{A}} / Z_{P}\right) m_{\mathrm{AWI}}$ (and $M_{\mathrm{PS}}^{2}$ ) is actually controlled by the strength of fermionic chiral breaking as measured by the parameter $\rho$ in front of $\mathcal{L}_{W}(2)$. An increase of $\rho$ by a factor 1.5 yields an increase of $\left(Z_{\tilde{A}} / Z_{P}\right) m_{\mathrm{AWI}}$ by a factor of about 2.4 (see Supplemental Material [25]) in good agreement with expectations from Ref. [20] and the above section Nambu-Goldstone phase and NP anomaly. For a correct interpretation of this finding one should notice that in more realistic models supporting the mass generation mechanism under discussion and accounting for EW interactions, parameters (like $\rho$ ) that control the strength of fermionic chiral breaking, and hence the NP mass terms, are not completely free, as they are constrained by the conditions of maximal restoration of the $\tilde{\chi}_{L} \times \tilde{\chi}_{R}$ symmetry (see Ref. [21] and Sec. VI of the Supplemental Material [25]).

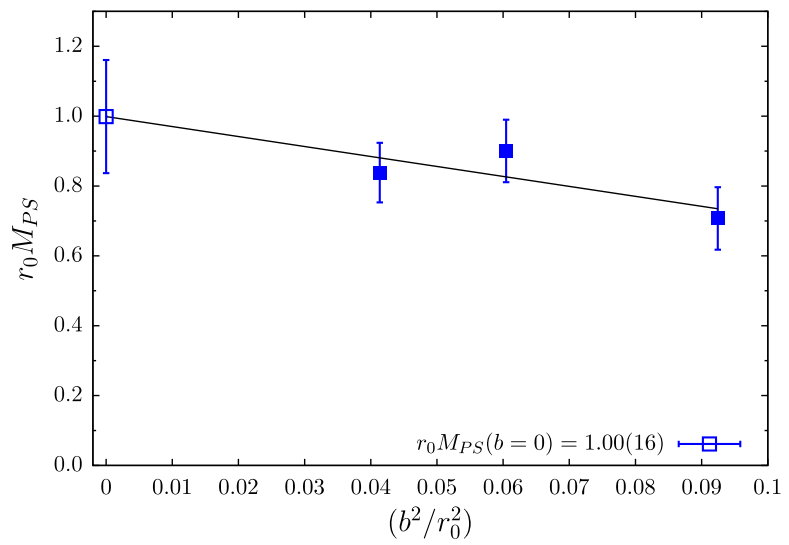

FIG. 3. $r_{0} M_{\mathrm{PS}}$ in the $\mathrm{NG}$ phase vs $\left(b / r_{0}\right)^{2}$ and its linear extrapolation to the continuum limit. 
Conclusions and outlook.-In this Letter by means of pioneering lattice simulations we have demonstrated from first principles the occurrence of an elementary fermion mass term as a NP anomaly in the EL of a renormalizable $\mathrm{SU}(3)$ gauge model [Eq. (1)]. In this model a doublet of strongly interacting fermions is coupled to a colorless complex scalar doublet via chiral breaking Yukawa and higher dimensional $\left[\mathcal{L}_{W}\right.$ in Eq. (2)] operators. Indeed, once bare parameters are chosen so as to ensure (maximal) restoration of fermionic chiral symmetries, a fermion mass of the order of the RGI scale $\left(\Lambda_{S}\right)$ is generated in the phase where the exact symmetry acting on fermions and scalars is spontaneously broken.

This result represents ground-breaking progress in quantum field theory as it provides evidence for the occurrence of a NP obstruction ("anomaly") to the recovery of broken fermionic chiral symmetries giving rise to a dynamically generated fermion mass term.

From a phenomenological viewpoint EW interactions can be included without introducing tree-level flavor changing neutral currents by promoting the exact $\chi_{L} \times$ $U(1)_{Y}$ invariance to a gauge symmetry. Since the $\tilde{\chi}_{L} \times \tilde{\chi}_{R}$ transformations now act both on fermions and weak bosons, one can show that (i) the requirement of maximal $\tilde{\chi}_{L}$ restoration leads to stringent constraints on the chiral breaking parameters and (ii) a unique NP mechanism generates fermion and weak boson mass terms [21].

This mass generation phenomenon is an alternative to the Higgs mechanism and provides an interesting starting point for beyond the SM models. As all masses are parametrically of the order of the RGI scale, the latter must be much larger than $\Lambda_{\mathrm{QCD}} \sim 300 \mathrm{MeV}$, if the masses of the top quark and the EW bosons have to be explained. This observation suggests the existence of a new non-Abelian gauge interaction that gets strong at a scale $\Lambda_{T} \gg \Lambda_{\mathrm{QCD}}$, and of new elementary fermions with $O\left(\Lambda_{T}\right)$ NP masses. Crude estimates [20,21] hint at $\Lambda_{T}=O(\mathrm{a}$ few TeV $)$. Since the condition of $\tilde{\chi}_{L}$ restoration implies the decoupling of $\zeta_{0}$ (the isosinglet component of $\Phi$ ), one ends up with models of the composite Higgs [15,16] type, where the $125 \mathrm{GeV}$ resonance is a bound state $[20,21]$ in the $W W+Z Z$ channel formed owing to the new strong force.

In the theoretical framework sketched above we see also a chance of understanding the observed fermion mass hierarchy. Denoting by $c_{1, f} \Lambda_{T}$ the dynamical mass of the SM fermion $f$ and by $g$ the gauge coupling of the strongest gauge interaction which $f$ is subjected to, it turns out [20] that $c_{1, f}$ is $O\left(g^{4}\right)$ for the heaviest fermion generation and possibly of higher order for the other generations. In this way one can understand, e.g., the top to $\tau$ mass ratio [21]. Further remarks about extending the model (1) to a phenomenologically sensible theory are deferred to the Supplemental Material [25].

In summary, by combining the condition of maximal restoration of fermionic chiral symmetry explicitly broken at the UV scale (a weak form of 't Hooft naturalness) with the assumption of the existence of a new non-Abelian gauge interaction with $\Lambda_{T}=O(\mathrm{a}$ few $\mathrm{TeV})$, we find a novel mechanism that gives mass to elementary fermions and EW gauge bosons. In models based on this mechanism the EW scale can be related to the scale of new physics at which new resonances should be detected in accelerator experiments.

We are grateful to J. Kuti, M. Lüscher, G. Martinelli, N. Tantalo, and M. Testa for very valuable discussions. We thank G. M. de Divitiis, B. Knippschild, and M. Schröck for their participation in the early stages of this project. CPU time on Galileo and Marconi clusters was provided by the INFN group LQCD123 under CINECA-INFN agreements. This work was supported in part by the DFG in the Sino-German research center CRC110 and by INFN under the project HPC_HTC

*Corresponding author. roberto.frezzotti@roma2.infn.it

[1] S. Weinberg, Phys. Rev. Lett. 19, 1264 (1967).

[2] G. 't Hooft, NATO Sci. Ser. B 59, 135 (1980).

[3] C. Froggatt and H. Nielsen, Nucl. Phys. B147, 277 (1979).

[4] H. Nilles, Phys. Rep. 110, 1 (1984).

[5] S. Weinberg, The Quantum Theory of Fields. Vol. 3: Supersymmetry (Cambridge University Press, Cambridge, England, 2013).

[6] M. Tanabashi et al. (Particle Data Group), Phys. Rev. D 98, 030001 (2018).

[7] S. Weinberg, Phys. Rev. D 13, 974 (1976); 19, 1277(A) (1979).

[8] L. Susskind, Phys. Rev. D 20, 2619 (1979).

[9] S. Dimopoulos and L. Susskind, Proceedings: International Conference on High Energy Physics, Geneva, Switzerland, 1979: In 2 volumes, Nucl. Phys. B155, 237 (1979).

[10] E. Eichten and K. Lane, Phys. Lett. 90B, 125 (1980).

[11] B. Holdom, Phys. Lett. 150B, 301 (1985).

[12] K. Yamawaki, M. Bando, and K.-i. Matumoto, Phys. Rev. Lett. 56, 1335 (1986).

[13] T. Appelquist, D. Karabali, and L. C. R. Wijewardhana, Phys. Rev. Lett. 57, 957 (1986).

[14] T. Appelquist and L. C. R. Wijewardhana, Phys. Rev. D 36, 568 (1987).

[15] D. B. Kaplan, Nucl. Phys. B365, 259 (1991).

[16] G. Panico and A. Wulzer, Lect. Notes Phys. 913, 1 (2016).

[17] N. Arkani-Hamed, S. Dimopoulos, and G. R. Dvali, Phys. Lett. B 429, 263 (1998).

[18] L. Randall and R. Sundrum, Phys. Rev. Lett. 83, 3370 (1999).

[19] T. Gherghetta and A. Pomarol, Nucl. Phys. B586, 141 (2000).

[20] R. Frezzotti and G. C. Rossi, Phys. Rev. D 92, 054505 (2015).

[21] R. Frezzotti and G. Rossi, Proc. Sci. LATTICE2018 (2018) 190.

[22] K. G. Wilson, Phys. Rev. D 10, 2445 (1974).

[23] L. H. Karsten and J. Smit, Nucl. Phys. B183, 103 (1981).

[24] M. Bochicchio, L. Maiani, G. Martinelli, G. C. Rossi, and M. Testa, Nucl. Phys. B262, 331 (1985). 
[25] See Supplemental Material at http://link.aps.org/ supplemental/10.1103/PhysRevLett.123.061802 for more details about lattice formulation, data analysis, further checks and remarks, which also includes Refs. [20,21,26-59].

[26] A. De and J. Shigemitsu, Nucl. Phys. B307, 376 (1988).

[27] D. B. Kaplan, Phys. Lett. B 288, 342 (1992).

[28] H. Neuberger, Phys. Lett. B 417, 141 (1998).

[29] H. Kluberg-Stern, A. Morel, O. Napoly, and B. Petersson, Nucl. Phys. B220, 447 (1983).

[30] A. Patel and S. R. Sharpe, Nucl. Phys. B395, 701 (1993).

[31] Y. Luo, Phys. Rev. D 55, 353 (1997).

[32] I. Montvay and G. Muenster, Quantum Fields on a Lattice, Cambridge Monographs on Mathematical Physics (Cambridge University Press, Cambridge, England, 1997), https://doi.org/10.1017/CBO9780511470783.

[33] R. Frezzotti, P. A. Grassi, S. Sint, and P. Weisz (ALPHA Collaboration), J. High Energy Phys. 08 (2001) 058.

[34] R. Frezzotti and G. C. Rossi, J. High Energy Phys. 08 (2004) 007.

[35] F. Butler, H. Chen, J. Sexton, A. Vaccarino, and D. Weingarten, Nucl. Phys. B430, 179 (1994).

[36] C. Bernard, T. Blum, C. Detar, S. Gottlieb, U. M. Heller, J. E. Hetrick, C. McNeile, K. Rummukainen, R. Sugar, and D. Toussaint (MILC Collaboration), Phys. Rev. Lett. 81, 3087 (1998).

[37] S. Aoki et al. (CP-PACS Collaboration), Phys. Rev. Lett. 84, 238 (2000).

[38] T. Blum et al., Phys. Rev. D 69, 074502 (2004).

[39] G. Veneziano, Nucl. Phys. B117, 519 (1976).

[40] A. Morel, J. Phys. II (France) 48, 1111 (1987).

[41] C. W. Bernard and M. F. L. Golterman, Phys. Rev. D 46, 853 (1992).

[42] M. Guagnelli, R. Sommer, and H. Wittig (ALPHA Collaboration), Nucl. Phys. B535, 389 (1998).

[43] S. Necco and R. Sommer, Nucl. Phys. B622, 328 (2002).
[44] J. Bulava, P. Gerhold, K. Jansen, J. Kallarackal, B. Knippschild, C. J. D. Lin, K.-I. Nagai, A. Nagy, and K. Ogawa, Adv. High Energy Phys. 2013, 875612 (2013).

[45] M. Goeckeler and H. Leutwyler, Nucl. Phys. B361, 392 (1991).

[46] S. R. Sharpe, Phys. Rev. D 46, 3146 (1992).

[47] N. Carrasco et al., Nucl. Phys. B887, 19 (2014).

[48] G. Colangelo, S. Durr, and C. Haefeli, Nucl. Phys. B721, 136 (2005).

[49] K. Symanzik, Nucl. Phys. B226, 187 (1983).

[50] K. Symanzik, Nucl. Phys. B226, 205 (1983).

[51] M. Luscher, S. Sint, R. Sommer, and P. Weisz, Nucl. Phys. B478, 365 (1996).

[52] K. Jansen, M. Papinutto, A. Shindler, C. Urbach, and I. Wetzorke (XLF Collaboration), Phys. Lett. B 619, 184 (2005).

[53] K. Jansen, M. Papinutto, A. Shindler, C. Urbach, and I. Wetzorke (XLF Collaboration), J. High Energy Phys. 09 (2005) 071.

[54] S. L. Glashow, arXiv:hep-ph/0504287.

[55] M. E. Peskin and T. Takeuchi, Phys. Rev. Lett. 65, 964 (1990).

[56] M. E. Peskin and T. Takeuchi, Phys. Rev. D 46, 381 (1992).

[57] F. Romero-Lopez, A. Rusetsky, and C. Urbach, Phys. Rev. D 98, 014503 (2018).

[58] B. W. Lee, C. Quigg, and H. B. Thacker, Phys. Rev. D 16, 1519 (1977).

[59] R. Frezzotti, M. Garofalo, and G. C. Rossi, Phys. Rev. D 93, 105030 (2016).

[60] J. F. Donoghue, E. Golowich, and B. R. Holstein, Dynamics of The Standard Model (Cambridge University Press, Cambridge, England, 2014).

[61] C. Gattringer and C. B. Lang, Quantum Chromodynamics on the Lattice, Lecture Notes in Physics Vol. 788 (Springer, New York, 2010), pp. 1-343. 